\title{
Oxychlorination of Ethylene with Copper Chloride Catalyst
}

\author{
Naoya Kominami**, Kusuo Kawarazaki**, Yoshiaki Yamazaki** \\ Tokio Sakurai**
}

\begin{abstract}
Summary: In the oxychlorination of $\mathrm{C}_{2} \mathrm{H}_{4}$, copper chloride on the active carbon has a higher activity than catalysts on other sorts of carrier. Examinations were made as to what relations exist between the selectivity of 1, 2-dichloroethane and composition of supplied gas and the reaction temperature. Two ranges in which the selectivity of 1, 2-dichloroethane does not vary and $\mathrm{CO}_{2}$ produced in large quantity were observed. The breaking point of both range depends on the mole ratio of $\mathrm{HCl} / \mathrm{C}_{2} \mathrm{H}_{4}$ in the supplied gas and the reaction temperature. When the reaction is performed with the supplied gas having higher than 2 mole ratio of $\mathrm{HCl} / \mathrm{C}_{2} \mathrm{H}_{4}$, the oxide-forming range does not appear. The forming rate of 1,2-dichloroethane is shown by the following equation at chloride-forming range,
\end{abstract}

$$
r=k P_{C_{2} H_{4}} P_{H C l^{0.2}} .
$$

Considering the rate equation shown above, the change of catalytic state and the results of the pulse technique, it is reduced that the mechanism of the oxychlorination of $\mathrm{C}_{2} \mathrm{H}_{4}$ with copper chloride catalyst is consisted of the following two equations,

$$
\begin{aligned}
& \mathrm{C}_{2} \mathrm{H}_{4}+2 \mathrm{CuCl} 2 \longrightarrow \mathrm{C}_{2} \mathrm{H}_{4} \mathrm{Cl}_{2}+\mathrm{Cu}_{2} \mathrm{Cl}_{2} \\
& \mathrm{Cu}_{2} \mathrm{Cl}_{2}+2 \mathrm{HCl}+\frac{1}{2} \mathrm{O}_{2} \longrightarrow 2 \mathrm{CuCl}_{2}+\mathrm{H}_{2} \mathrm{O}
\end{aligned}
$$

and the former is rate determining step.

\section{Introduction}

As to synthesis of 1,2-dichloroethane there are many known methods using copper chloride; for example, a method in which copper chloride is used as a chlorinating reagent and the reduced copper chloride converted into the original copper chloride by hydrogen chloride and oxygen to be used again $^{1 \prime}$ and a method in which 1,2-dichloroethane is derived from ethylene, hydrogen chloride and oxygen with copper chloride as a catalyst ${ }^{2}$. These methods, however, require high reaction temperatures, so if there can be obtained a high activity at lower reaction temperatures, it is more advantageous, commercially, from the standpoints of materials of reactors, removal of heat, capacity of apparatus and prevention of byproducts.

* Received December 1, 1965.

** Research Laboratory of Technology, Asahi Chemical Industry Co., Ltd., 3-27-36, Nakadai-cho, Itabashi-ku, Tokyo.
The authors have found that a high activity at low temperatures can be obtained by using an active carbon carrier, and also obtained some knowledge about the mechanism of the said catalyst, which are reported hereinafter.

\section{Experimental}

\section{Specimen}

The used ethylene, oxygen, nitrogen and hydrogen chloride were obtained on the market, packed in cylinders.

Catalyst

100 grams of active chrbon (Sirasagi C of Takeda Yakuhin Co., Ltd.) which had been washed thoroughly with acid and then with water and dried, were immersed in an aqueous solution of $50 \mathrm{~m}$. moles of copper chloride, and after evaporating it to dryness on a water bath, it was used in the reaction. The catalysts of silica gel and alumina carriers were prepared similarly to the catalyst of active carbon, with commercially obtained 


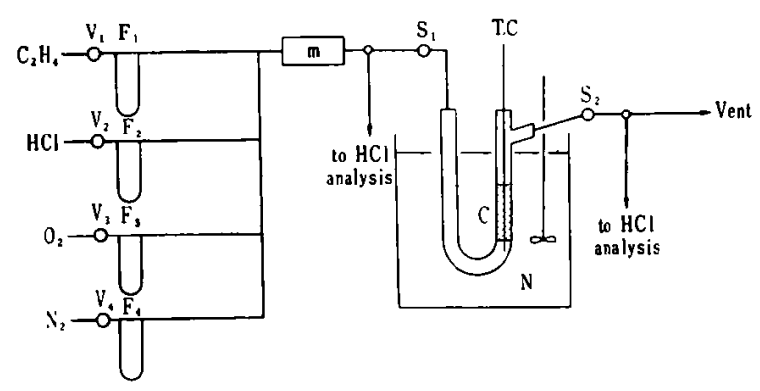

$V_{1}, V_{2}, V_{3}, V_{4}$ : Needle valve

$\mathrm{F}_{1}, \mathrm{~F}_{2}, \mathrm{~F}_{3}, \mathrm{~F}_{4}$ : Flow meter

$\mathrm{S}_{1}, \mathrm{~S}_{2} \quad$ : Sampling outlet

T.C : Thermo couple

$\mathrm{m} \quad$ : Mixer

C : Catalyst layer

N : Nitre bath

Fig. 1 Apparatus

obtained silica gel and $r$-alumina.

\section{Apparatus}

The ordinary following-type reactor shown in Fig. 1 was employed. 3.0 c.c. of the catalyst diluted with 12 c.c. of quarts sand were filled in a U-type reaction tube of pyrex glass having the inner diameter of about $10 \mathrm{~mm}$. It was heated by a nitre bath. Any difference between the temperatures of the nitre bath and the catalyst layer in the reaction tube was hardly observed.

\section{Analysis}

All samples were analysed by gas chromatography except for the analysis of hydrogen chloride which was by iodometry. The flowing rate was calibrated based on nitrogen as the inner standard.

\section{Results and Discussion}

\section{Influences of Carriers}

Experiments with various carriers were performed to clarify the effect of various carriers, and the results are shown in Table 1. The catalyst of the activated charcoal carrier showed much higher activity of pro-

Table 1 Influences of Carriers

\begin{tabular}{l|c|c|c}
\hline Sorts of carriers & $\begin{array}{c}\text { Conversion } \\
\text { of } \mathrm{C}_{2} \mathrm{H}_{4}\end{array}$ & $\begin{array}{c}\text { Selectivity } \\
\text { of } \\
1,2 \text {-dichloro- } \\
\text { ethane }\end{array}$ & $\begin{array}{c}\text { BET } \\
\text { surface }\end{array}$ \\
\hline Active carbon & $15.5 \%$ & $98.0 \%$ & $745 \mathrm{~m}^{2} / \mathrm{g}$ \\
Silica gel & 4.8 & 96.8 & 470 \\
$\gamma \cdot \mathrm{Al}_{2} \mathrm{O}_{3}$ & 6.4 & 98.5 & 390 \\
\hline
\end{tabular}

$\mathrm{CuCl}_{2} \quad 70 \mathrm{~m}$ mole $/ 100 \mathrm{~g}$ carrier

Temp. $150^{\circ} \mathrm{C}$ SV $150 \mathrm{hr}^{-1}$

$\mathrm{C}_{2} \mathrm{H}_{4}: \mathrm{HCl}: \mathrm{O}_{2}=4: 1: 1$ ducing 1,2-dichloroethane than the other two catalysts. The high selectivity of 1,2dichloroethane against the converted ethylene was found with each carrier, and no difference was observed on the selectivity due to the catalysts. The third column of the table shows the surface area of each carrier measured by BET method. As clearly seen in the table, a co-relationship between the surface area of the carrier and the activity is hardly recognized, so the difference on the activity cannot be considered simply as the difference of the surface area of the carrier. It was found, particularly, that very high activity could be obtained by using the active carbon carrier.

Factors for the Selectivity of 1,2-dichloroethane

The relations of the selectivity of 1,2 dichloroethane to the composition of supplied gas and to the reaction temperature, with the catalyst of the active carbon carrier, are shown in Fig. 2. The conversion of

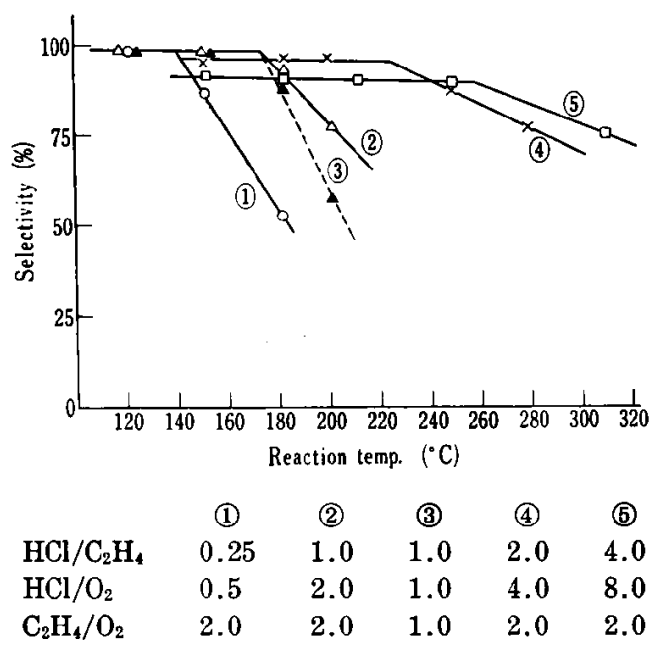

Fig. 2 Selectivity of 1, 2-dichloroethane

ethylene was, of course, increased by raising the reaction temperature, but this relation is not shown in Fig. 2: Relations of the reaction temperature to the conversion of ethylene and the one-pass yields of 1,2dichloroethane and carbon dioxide are shown in Fig. 3 in which the molar ratio of the supplied gas was $\mathrm{HCl}: \mathrm{C}_{2} \mathrm{H}_{4}: \mathrm{O}_{2}=2: 2: 1$. According to these results, the following can be pointed out:

i) With the supplied gas having respective molar ratio, the curve of the selectivity of 


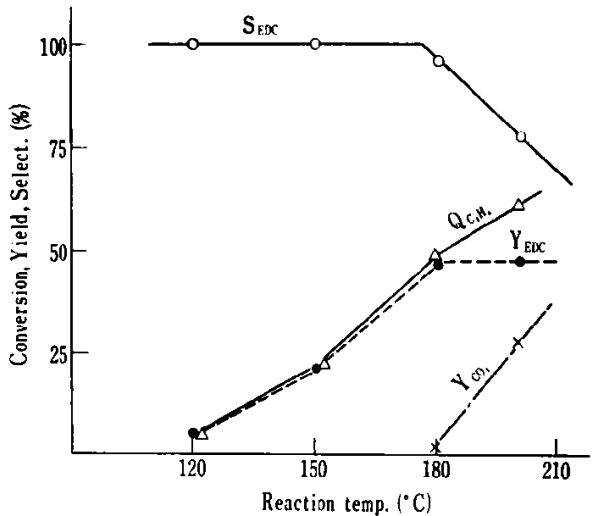

$\mathrm{HCl}: \mathrm{C}_{2} \mathrm{H}_{4}: \mathrm{O}_{2}=2: 2: 1$

Fig. 3 Temperature dependency

1,2-dichloroethane in the figure shows a breaking point at the reaction temperature, and the point divides the range into two parts: one is the range in which the selectivity of 1,2-dichloroethane is not varied and the curve is flat, and 1,2-dichloroethane is the main product; this range is named as the chloride-forming range. The other is the range in which the selectivity of 1,2-dichloroethane is steeply sloped down with the raising of the reaction temperature, and the selectivity of carbon dioxide and carbon monoxide is increased: this range is named as the oxide-forming range.

ii) As clearly seen in Fig. 3, the conversion of ethylene is increased with the rise in the temperature. However, though the one-pass yield of 1,2-dichloroethane is increased similarly to the conversion ratio of ethylene, the one-pass yield of 1,2-dichloroethane is hardly increased at temperatures higher than $180^{\circ} \mathrm{C}$. At the reaction temperature of $180^{\circ} \mathrm{C}$ carbon dioxide begins to form, and its one-pass yield almost coincided with twice the increased value of the conversion of ethylene: that is, formation of carbon dioxide and carbon monoxide in the oxideforming range is due to oxidation of ethylene of the raw material, but not to the combustion of active carbon used as a carrier.

iii) The said breaking point is transferred to the higher temperature side with the increase of the molar ratio of $\mathrm{HCl} / \mathrm{C}_{2} \mathrm{H}_{4}$ in the supplied gas; that is, the position of the breaking point is determined by the molar ratio of $\mathrm{HCl} / \mathrm{C}_{2} \mathrm{H}_{4}$ in the supplied gas.

iv) The descending slope of the selectivity of 1,2-dichloroethane in the oxide-forming range is influenced by the molar ratio of $\mathrm{HCl} / \mathrm{C}_{2} \mathrm{H}_{4}$ in the supplied gas.

v) The selectivity of 1,2-dichloroethane in the chloride-forming range is gradually decreased with the increase of the molar ratio of $\mathrm{HCl} / \mathrm{C}_{2} \mathrm{H}_{4}$ in the supplied gas. It is due to the increase of the conversion ratio of ethyl chloride.

vi) When the reaction is performed with the supplied gas having higher than 2 molar ratio of $\mathrm{HCl} / \mathrm{C}_{2} \mathrm{H}_{4}$, which is the stoichiometric molar ratio according to the present reaction, no oxide is formed. Though the breaking point is found on the curve (5) in Fig. 2, in the descending range in the right side of the point, the consecutive reaction product of 1,2-dichloroethane or vinyl chloride is increased, and the selectivity of 1,2-dichloroethane is decreased apparently. 1,1,2-trichloroethane and 1,2-dichloroethylene are main consecutive reaction products.

Influences of Space Velocity and Reaction Temperature

The reaction was carried out by varying the space velocity at each reaction temperature and the results are shown in Fig. 4 and Fig. 5. Fig. 4 and Fig. 5 show the re-

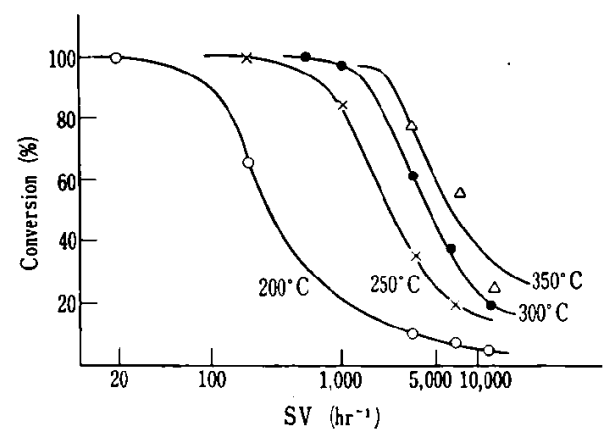

Fig. 4 Ethylene conversion

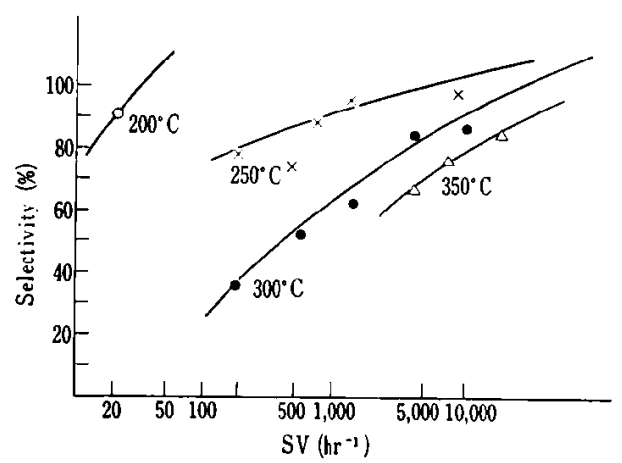

Fig. 5 Selectivity of 1,2-dichloroethane 
lations of the conversion of ethylene and of the selectivity of 1,2-dichloroethane to the space velocity and the temperature, respectively. From these figures, it is found that by increasing the space velocity, the conversion of ethylene is decreased in each case, but the selectivity of 1,2-dichloroethane is increased. Then these results are summarized as shown in Fig. 6, in which the

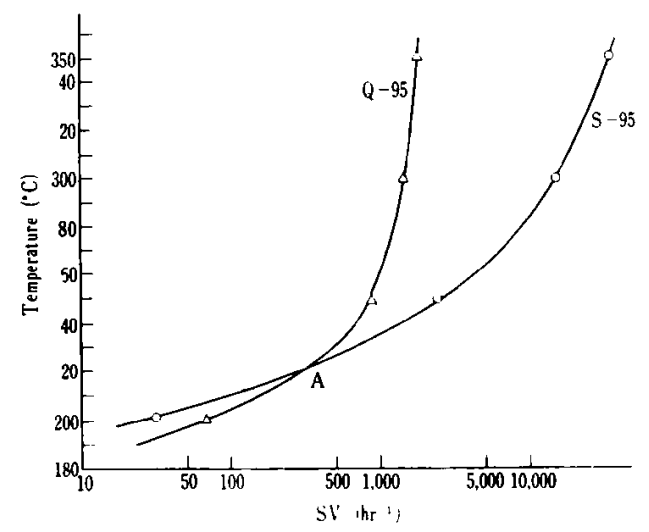

Fig. 6 Specific diagram on relation between conversion and space velocity

ordinate is the reaction temperature and the abscissa is the logarithm of the space velocity. The curve of Q-95 in the figure shows the relation of the reaction temperature to the space velocity on $95 \%$ of the conversion of ethylene being obtained, and with the conditions above the curve the conversion of ethylene is higher than $95^{\circ} \%$. The curve of S-95 shows the relation of the reaction temperature to the space velocity to obtain $95 \%$ of the selectivity of 1,2-dichloroethane and with the conditions under the curve the selectivity of 1,2-dichloroethane is higher than $95 \%$. Therefore, with the conditions within the range enclosed with the said two curves, the one-pass yield of 1,2-dichloroethane is higher than $90.3 \%$. The maximum effect of the catalyst to obtain $90.3 \%$ of the one-pass yield of 1,2-dichloroethane is obtained with the condition shown by the cross point of the said two curves. (Point $\mathrm{A}$ in the figure.)

\section{Reaction Mechanism}

To the present catalyst, the gas mixture having the $\mathrm{HCl} / \mathrm{C}_{2} \mathrm{H}_{4}$ molar ratio from 2 to 4 was fed at $300^{\circ} \mathrm{C}$ with the space velocity of $1,500 \mathrm{hr}^{-1}$, and chlorine was not recognized at the outlet. The equilibrium in Deacon reaction deviates to the side of the product system at temperatures near $300^{\circ} \mathrm{C}^{3}$; therefore, it is assumed that oxychlorination with the present catalyst is not progressed via Deacon reaction.

When introducing ethylene over the present catalyst, 1,2-dichloroethane is produced and cupric chloride is reduced to cuprous chloride. When feeding only hydrogen chloride or oxygen over the catalyst of which 1,2dichloroethane forming activity has been lost due to almost all of cupric chloride having been converted to cuprous chloride, 1,2dichloroethane forming activity is not recovered, but on feeding the gas mixture of hydrogen chloride and oxygen, the reactivity is recovered and, in that case, the catalyst is changed to cupric chloride.

The effect of the partial pressures of the components on the reaction rate was studied by a series of experiments by varying the partial pressure of one of the components of the gas, that is, ethylene, hydrogen chloride and oxygen, while keeping the partial pressure of the other components constant by nitrogen-diluting method. The reaction was performed at $175^{\circ} \mathrm{C}$ with the space velocity of $1,500 \mathrm{hr}^{-1}$ with the molar ratio of the supplied gas in the chloride-forming range; and it was found that the forming rate of 1,2-dichloroethane was dependent solely on the partial pressure of ethylene.

$$
\frac{d E D C}{d t}=k P_{\mathrm{C}_{2} \mathrm{H}_{4}} \cdot P_{\mathrm{HCl}^{0.2}}
$$

The catalyst during the said reaction remained almost in the form of cupric chloride.

According to the results described above, it is considered that oxychlorination of ethylene is consisted of two reactions shown by equations (a) and (b), and in the case of the copper chloride catalyst, the reaction of (a), that is, the reaction of ethylene with copper chloride, is the rate determining step.

$$
\begin{aligned}
& \mathrm{C}_{2} \mathrm{H}_{4}+\mathrm{CuCl}_{2} \longrightarrow \mathrm{C}_{2} \mathrm{H}_{4} \mathrm{Cl}_{2}+\mathrm{CuCl}_{2-x} \\
& \mathrm{CuCl}_{2-x}+\mathrm{HCl}+\mathrm{O}_{2} \longrightarrow \mathrm{CuCl}_{2}+\mathrm{H}_{2} \mathrm{O}
\end{aligned}
$$

\section{References}

1) U.S. $2,752,402 ; 2,407,828$ etc.

2) Brit. 781,$414 ; 2,783,286$ etc.

3) Arnold, C.W., Kobe, K. A., Chem. Eng. Process, 48, 293 (1952). 